\section{Asbestos: Avoiding a Pyrrhic Victory}

The recent announcement by the newly elected Quebec Government that it would withdraw its loan guarantee to the Jeffrey asbestos mine, and the subsequent announcement by the Federal Government that it would drop its objection to the inclusion of chrysotile asbestos on the United Nations' Rotterdam Convention, constitute a major public health victory.

Although Canada's contribution to the worldwide production of asbestos is less than 20\% (compared to Russia's 50\%), its international standing gave its position on chrysotile credibility and justified other countries' continued production and export of the deadly fibre. Our country's position that chrysotile was not harmful was particularly hypocritical given the incontrovertible evidence and the fact that our government was actively removing asbestos from public buildings.

Canada's assertion that chrysotile could be safely handled with proper protections, while exporting the fibre to countries with no or ineffective workers' safety regulations, was indefensible and unethical and it led to a campaign by public health professionals and activists to pressure governments to abandon their support for the asbestos industry.

The governments' recent change of heart is good news for all except for the mining communities which profited from the local jobs created by the industry. These communities now face the critical task of renewing their employment base to insure their survival. Herein lies a new challenge for Canadian public health. As we enter more systematically and forcefully into the public policy arena, we have a moral responsibility to all those affected by our actions. In this case, we are now collectively accountable to the former mining communities to ensure that the ultimate outcome is favourable. It would be hypocritical for us to claim victory for saving lives of workers in countries that imported Canadian chrysotile while ignoring the plight of the workers of Quebec mining towns. Public health professionals unfortunately have little if any training and expertise in economic development or in many other sectors which collectively form what we call the social determinants of health.

We can name these determinants, we try to influence them, but most of them are beyond our control and we have a very poor understanding of the dynamic processes that affect these determinants or of the intended and unintended outcomes of policy changes. Political and economic sciences are critical to building effective public health interventions and to effecting policy changes and managing consequences of our actions. Close partnerships with these and other disciplines are an imperative if we want public health practice to be effective over the next decade.

We neglect the potential negative consequences of interventions at our own risk. Let us not transform our public health battles into pyrrhic victories.

Gilles Paradis

Scientific Editor

\section{Amiante : évitons une victoire à la Pyrrhus}

L'annonce récente par le gouvernement nouvellement élu du Québec du retrait de sa garantie de prêt à la mine d'amiante Jeffrey et l'annonce subséquente par le gouvernement fédéral de l'abandon de son objection à l'inclusion de l'amiante chrysotile dans la Convention de Rotterdam des Nations Unies constituent une grande victoire pour la santé publique.

Bien que la part du Canada dans la production mondiale d'amiante soit inférieure à $20 \%$ (contre $50 \%$ pour la Russie), le prestige international du Canada conférait une crédibilité à sa position sur le chrysotile et justifiait dans d'autres pays le maintien de la production et de l'exportation de cette fibre mortelle. L'argumentation canadienne sur l'innocuité du chrysotile était particulièrement hypocrite devant les preuves incontestables du contraire et le fait que notre gouvernement s'employait activement à retirer l'amiante de ses bâtiments publics.

Le Canada affirmait que le chrysotile pouvait être manipulé sans danger avec des mesures de protection adéquates, tout en l'exportant dans des pays où la réglementation de la sécurité des travailleurs était inefficace ou inexistante; c'était une affirmation indéfendable et contraire à l'éthique; elle a poussé les militants et les professionnels de la santé publique à faire campagne pour que les gouvernements abandonnent leur appui à l'industrie de l'amiante.

Le récent changement d'avis des gouvernements est une bonne nouvelle pour tout le monde, sauf pour les communautés minières qui ont profité des emplois locaux créés par l'industrie. Ces communautés ont maintenant la tâche cruciale de renouveler leur base d'emploi pour assurer leur survie. C'est là un nouveau défi pour la santé publique canadienne. Dès que nous entrons systématiquement et vigoureusement dans l'arène des politiques publiques, nous avons une responsabilité morale envers toutes les personnes touchées par nos actions. Dans ce cas-ci, nous avons maintenant la responsabilité collective envers les anciennes communautés minières de faire en sorte que le résultat final leur soit favorable. Il serait hypocrite de crier victoire pour avoir sauvé les vies de travailleurs dans les pays qui importaient le chrysotile canadien tout en fermant les yeux sur le sort des ouvriers des villes minières du Québec. Les professionnels de la santé publique ont malheureusement très peu de formation et d'expertise en développement économique et dans les nombreux autres domaines qui englobent ce que nous appelons les déterminants sociaux de la santé.

Nous pouvons nommer ces déterminants, nous essayons de les influencer, mais la plupart sont hors de notre contrôle; nous connaissons très mal la dynamique qui joue sur ces déterminants, et les résultats attendus et inattendus des changements de politique. Les sciences politiques et économiques sont essentielles à l'élaboration d'interventions de santé publique efficaces, à l'avènement de changements de politique et à la gestion des conséquences de nos actions. Il est donc impératif de tisser des partenariats étroits avec ces deux disciplines, et plusieurs autres, si nous voulons que la pratique de la santé publique soit efficace au cours de la prochaine décennie.

Si nous négligeons les éventuelles conséquences négatives de nos interventions, ce sera à nos risques et périls. Ne transformons pas nos batailles pour la santé publique en victoires à la Pyrrhus.

Le rédacteur scientifique, Gilles Paradis 